Impact of substrate surface scratches on the laser damage resistance of multilayer coatings

S. Qiu, J. Wolfe, A. Monterrosa, N. Teslich, M. Feit, T. Pistor, C. Stolz

November 15, 2010

SPIE Laser Damage Conference Boulder, CO, United States

September 26, 2010 through September 29, 2010 
This document was prepared as an account of work sponsored by an agency of the United States government. Neither the United States government nor Lawrence Livermore National Security, LLC, nor any of their employees makes any warranty, expressed or implied, or assumes any legal liability or responsibility for the accuracy, completeness, or usefulness of any information, apparatus, product, or process disclosed, or represents that its use would not infringe privately owned rights. Reference herein to any specific commercial product, process, or service by trade name, trademark, manufacturer, or otherwise does not necessarily constitute or imply its endorsement, recommendation, or favoring by the United States government or Lawrence Livermore National Security, LLC. The views and opinions of authors expressed herein do not necessarily state or reflect those of the United States government or Lawrence Livermore National Security, LLC, and shall not be used for advertising or product endorsement purposes. 


\title{
Impact of substrate surface scratches on the laser damage resistance of multilayer coatings
}

\author{
S. Roger Qiu ${ }^{a^{*}}$, Justin E. Wolfe ${ }^{a}$, Anthony M. Monterrosa ${ }^{b}$, William A. Steele ${ }^{a}$, Nick E. \\ Teslich, Michael D. Feit ${ }^{\mathrm{a}}$, Thomas V. Pistor ${ }^{\mathrm{c}}$, Christopher J. Stolz ${ }^{\mathrm{a}}$ \\ ${ }^{a}$ Lawrence Livermore National Laboratory, 7000 East Avenue, Livermore, CA 94551 \\ ${ }^{b}$ Department of Nuclear Engineering and Department of Materials Science \& Engineering, \\ University of California, Berkeley, CA 94704 \\ ${ }^{c}$ Panoramic Technology Inc., 2039 Shattuck Ave., Suite 404, Berkeley, CA 94704 \\ Phone: (925) 422-1636, FAX: (925) 423-0792, email: qiu2@llnl.gov
}

\begin{abstract}
Substrate scratches can limit the laser resistance of multilayer mirror coatings on high-peak-power laser systems. To date, the mechanism by which substrate surface defects affect the performance of coating layers under high power laser irradiation is not well defined. In this study, we combine experimental approaches with theoretical simulations to delineate the correlation between laser damage resistance of coating layers and the physical properties of the substrate surface defects including scratches. A focused ion beam technique is used to reveal the morphological evolution of coating layers on surface scratches. Preliminary results show that coating layers initially follow the trench morphology on the substrate surface, and as the thickness increases, gradually overcoat voids and planarize the surface. Simulations of the electrical-field distribution of the defective layers using the finite-difference timedomain (FDTD) method show that field intensification exists mostly near the top surface region of the coating near convex focusing structures. The light intensification could be responsible for the reduced damage threshold. Damage testing under $1064 \mathrm{~nm}, 3$ ns laser irradiation over coating layers on substrates with designed scratches show that damage probability and threshold of the multilayer depend on substrate scratch density and width. Our preliminary results show that damage occurs on the region of the coating where substrate scratches reside and etching of the substrate before coating does not seem to improve the laser damage resistance.
\end{abstract}

Keywords: Laser-induced damage, substrate scratch, light intensification, FIB, FDTD, multilayer coating.

\section{INTRODUCTION}

High dielectric constant electron-beam deposited multilayer coatings composed of silica and hafnia are frequently used on large-aperture mirrors for high peak power laser systems. For a robust laser system, high laser resistance is an obvious performance requirement. Defective features in the multilayer coatings as well as on the substrate surface are believed to play important roles in limiting mirror function and lifetime. For example, features such as highly-absorbing nano-clusters near the top surface of the coating, or solid inclusions buried deep within the multilayer coating, although rare, can result in damage upon laser light exposure.[1-2] Moreover, a recent study has shown that substrate scratches and impurities generated from surface finishing of the BK7 substrate can also lead to laser-induced damage on the multilayer coating.[3-4] Substrate scratches on the optic surface are inevitably created during the final shaping of the component through abrasive cutting, grinding and final polishing. Earlier studies have shown that substrate scratches on fused silica substrates act as precursors and lead to laser damage by inducing sub-bandgap absorption [5-6] which can be reduced by chemical etching of the substrate to improve optic performance. It is uncertain if the lessons learned from transparent optics is applicable to mirrors. To understand the impact of substrate scratches on the damage resistance of mirror coatings, we examined the damage threshold of silica-hafnia multilayer mirror coatings deposited on BK7 substrates with pre-engineered scratches of different widths. Our preliminary results show that damage does occur first in the film where scratches reside. Etching of the substrate before coating, however, does not appear to improve the laser damage resistance of the coating. 


\section{EXPERIMENTAL METHOD}

A series of six polished $50 \mathrm{~mm}$ diameter BK7 optics were used in this study. Scratch widths ranging from 15-30 $\mu \mathrm{m}$, 30-45 $\mu \mathrm{m}$, and 45-60 $\mu \mathrm{m}$ were created on two sets of uncoated samples, respectively; with one of each sample etched and the sister sample remaining unetched. The scratches were created by dragging the sample surface across a pad that contained imbedded silica spheres of $200 \mu \mathrm{m}, 300 \mu \mathrm{m}$ or $400 \mu \mathrm{m}$, diameter depending on the desired scratch width (see Table 1). The load placed on each sample was 4 pounds. To etch the scratches, three samples of different scratch width were dipped in a BOE (6:1) solution for 30 minutes followed by a deionized water wash and air dry.

\begin{tabular}{|c|c|}
\hline Scratch width $(\mu \mathrm{m})$ & Size of silica bead applied $(\mu \mathrm{m})$ \\
\hline $15-30$ & 200 \\
\hline $30-45$ & 300 \\
\hline $45-60$ & 400 \\
\hline
\end{tabular}

Table 1. Size of silica bead used for creating scratches of different width

Both etched and non-etched samples were then coated with a high reflector at $1064 \mathrm{~nm}$ consisting of multilayers of silica and hafnia dielectric materials with quarter-wave thicknesses. The coated samples were tested for laser damage resistance by following the damage test protocol given in ref. [7]. Briefly, a $1064 \mathrm{~nm}, 3 \mathrm{~ns}$ laser with a 1 mm Gaussian spot at $1 / \mathrm{e}^{2}$ is raster scanned in approximately $200 \mu \mathrm{m}$ steps across the test area of $1 \mathrm{~cm}^{2}$, starting at a low fluence and stepping up in 2 or $3 \mathrm{~J} / \mathrm{cm}^{2}$ increments until damage is observed. The small increment between laser shots is to maximize the fluence uniformity across the $1 \mathrm{~cm}^{2}$ test area. The damage fluence is defined as the lowest fluence at which any growing sites are observed or when more than 10 stable pinpoint sites are observed.

To evaluate electric-field intensification within the multilayer coating at the presence of substrate scratches, a commercially available software code TEMPESTpr2 [8-9] was utilized. TEMPESTpr2 employs a finite-difference time-domain (FDTD) method [10] to solve Maxwell's equations within a particular structure, both in 2-D and 3-D. The algorithm for this study simulates the scattering of an electromagnetic plane wave within the multilayer mirror topography containing a distorted region due to the existence of the substrate scratch. The simulation domain is rectangular, 2-D, and gridded uniformly. In the original code, the periodic boundary conditions (PBC) are applied in the x-direction, while the Berenger's perfectly matched layer (PML) absorbing-boundary condition [11] is applied in the vertical directions. Absorbing layers with median refractive indices were added in the outside edges of the simulation domain in the $\mathrm{z}$ direction to minimize the impact of the $\mathrm{PBC}$ and to prevent back reflections. A similar approach has been successfully used to quantify the electrical field intensification within a multilayer mirror design at the presence of conical pits. [12-13]

The structural feature of the defective multilayer used for the simulation was revealed by cross sectioning the region where the substrate scratch resides. The cross-sectional view of the interested region was achieved by trenching the coating materials at a $45^{\circ}$ angle with respect to the surface normal using a focused beam of gallium ions [14] in a high vacuum chamber. Because of the dielectric nature of the multilayer, the sample surface was coated with a thin layer of Au before cutting to prevent sample charging. The morphology of the cross-sectioned region was then characterized by a scanning electron microscope in the same chamber. Before imaging, a thin layer of $\mathrm{Pd}(<10 \mathrm{~nm})$ was coated on the freshly milled area to remove any charging effects..

\section{RESULTS AND DISCUSSION}

Similar to transparent optics such as fused silica, multiple types of scratches are often found on the BK7 substrate surface after final polishing. The scratches can be a sleek due to plastic abrasive wear, trailing indent from brittle fracture, or a mixture of both (trailing indent with lateral fracture). Examples of these scratches on a BK7 surface are shown in Figure 1. The image shown in Figure 1 is collected after the surface has been coated with a high 
reflector multilayer. Because the multilayer coating is thin and transparent in the visible spectrum, the scratches underneath the film can be easily discerned under an optical microscope.

The existence of scratches on the substrate surface can affect the morphology of the multilayer coating. The alteration of the coating topography can be quite significant if the scratches have complicated structures. Figure 2(a) is a SEM image showing a cross-sectional view of the multilayer coating of alternating hafnia and silica layers deposited on a substrate with a trailing indent scratch. Because the substrate area is heavily deformed, the resulting coating layers lose their planar nature. It can be seen that when the first coating layer was deposited, it actually followed the contour of the defective structure. The deposited coating also shows the ability to self-heal during the coating process. This is quite clear on the portion of the coating that was deposited on the right trench of the scratch (see encircled area). For the first fifteen layers during deposition, each layer follows the morphology of the trench. At the same time, the materials tend to grow towards the middle of the void from both directions. Starting at the sixteenth layer, the dielectric layers from either side of the trench coalesce to form a continuous layer, but with a wedged geometry. As the coating layer number increases, the wedge gradually decreases and the coating is nearly planarized when the last layer is deposited.

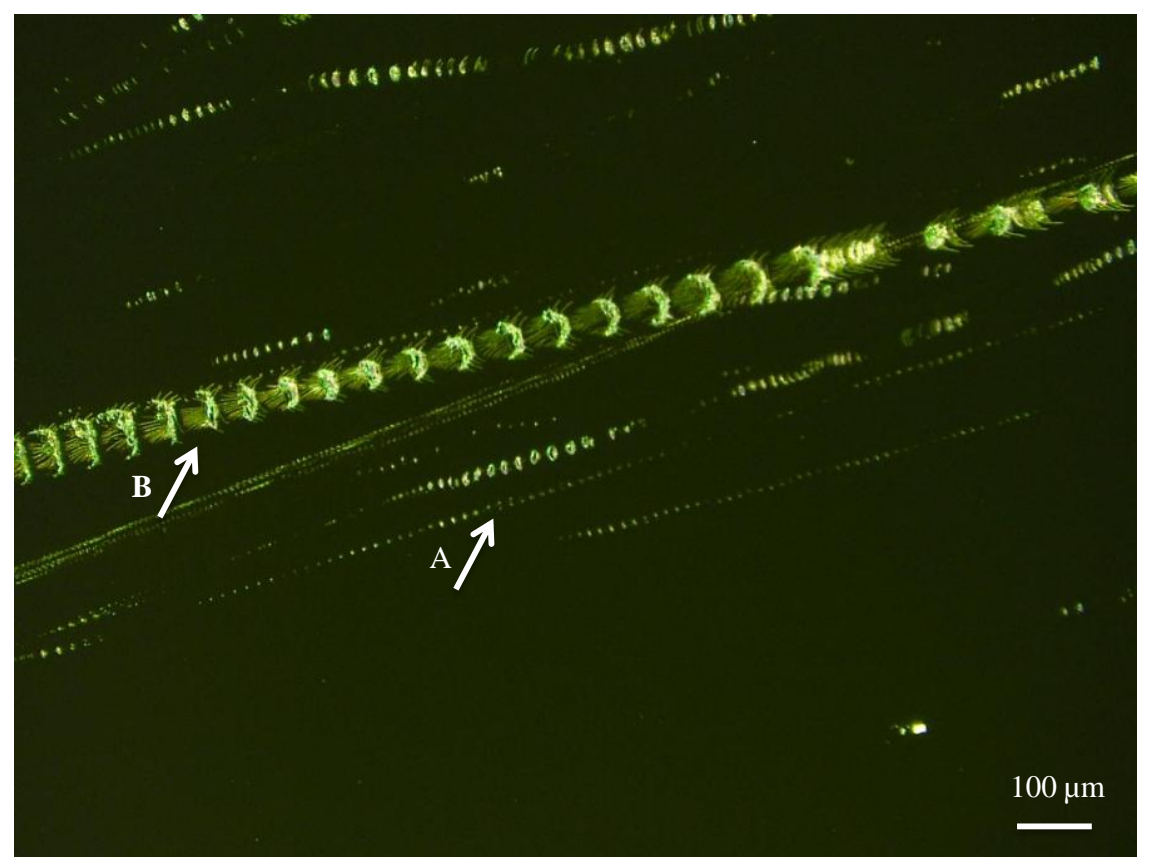

Figure 1. Optical microscope image shows the example of scratches including trailing indent (A) and trailing indent with lateral fracture (B) on the BK7 substrate.

The morphological alteration of the multilayer coating by a sleek type of scratch on the BK7 substrate is much less drastic. The coating can self-heal over less thickness and completely recover its flatness during the coating run if the sleek is small and shallow. Figure 2(b) is the cross-sectional view of a $1 \mu \mathrm{m}$ wide substrate sleek overcoated with a multilayer high reflector. Similar to that shown in Figure 2(a), the dielectric coating materials conform to the surface morphology including that of the scratch when it is first evaporated onto the substrate (see white box insert). The existence of the small sleek causes the coating layers to curve, but only at the first few layers. The coating achieves planarity by the middle of the coating run. Figure 2(c) shows the morphology of the multilayer coating deposited on another sleek which induced three ripples on the substrate surface. Although the resulting coating demonstrates a self-healing nature, it does not completely achieve planarity, thus forming a dome on the top layer. 

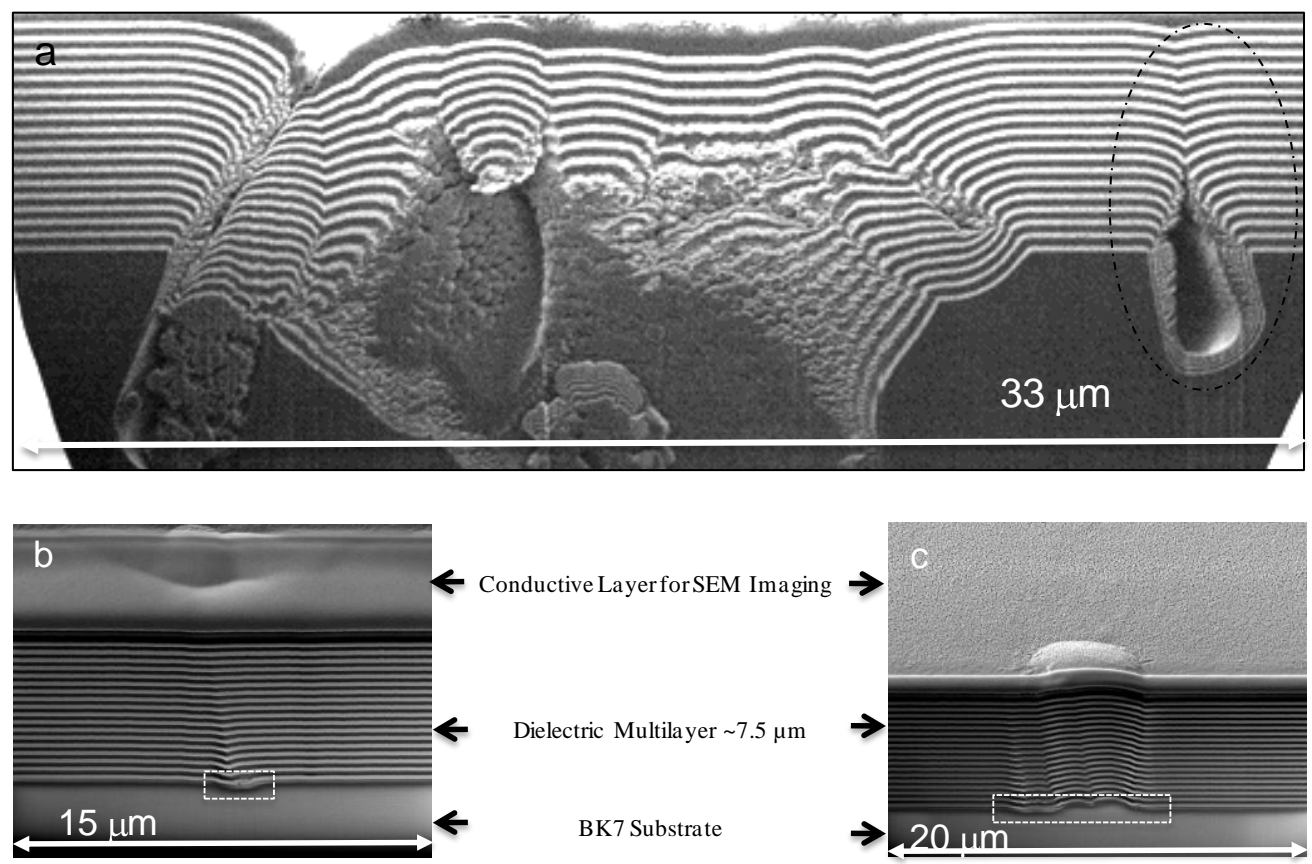

Figure 2. SEM images illustrating the cross-sectional view of coating layers on top of substrate scratches. (a) on an etched trailing indent type scratch, (b) on a un-etched, sleek type scratch, and (c) on an etched sleek type scratch.

The substrate scratch-induced perturbed multilayer coating can damage when the surface is exposed to laser light at a wavelength of $1064 \mathrm{~nm}$ and pulse width of $3 \mathrm{~ns}$. Damage testing following the NIF damage test protocol finds that initiation of the laser damage occurs frequently on the substrate scratches. Figure 3(a) shows the example of laserinduced damage sites on the coatings where the substrate scratches reside. The image was taken after the surface had been exposed to increasing higher fluences until damage growth was observed. As can be seen in the image, there is a size distribution to the damage sites and at the current resolution, nearly all of the discernable damage sites coincide with the substrate scratches. The damage site occurring in location A is most likely a growing site. Multiple sites are also observed on the same scratch, for example, location B.

To investigate the nature of the damage in more detail, high resolution images were taken. Figure 3 (b) is the magnified version of site B in Figure 3 (a). It can be clearly seen that laser-induced damage occurs first in the top layers and grows radially. Subsequent laser pulses then slowly begin damaging the next adjacent layer while continuing to radially grow the damaged top layers. Typically this type of damage has evidence of delamination of the silica layers and melting or boiling of the hafnia layers. Furthermore, stable and growing damage sites can occur on multiple spots of the same scratch. The radial damage growth can coalesce when damage sites are in close proximity and thus create a single damage site consisting of multiple initiation sites as shown in Figure 3 (b). The discoloration of the region near the scratch is likely caused by plasma scalding, re-deposition of damage debris, or depolarization induced by surface stresses.

The damage testing results for samples with different scratch widths are summarized in Figure 4. Typically the laser damage threshold of a non-defective section of the coating will exceed $100 \mathrm{~J} / \mathrm{cm}^{2}$. For non-etched substrates, the damage threshold is the same for all three scratch widths at $\sim 42 \mathrm{~J} / \mathrm{cm}^{2}$. The growth threshold, however, depends on the scratch width; the smaller the scratch width, the higher the fluence required for damage growth. For the scratch width smaller than $20 \mu \mathrm{m}$, no damage growth was observed at fluences exceeding $60 \mathrm{~J} / \mathrm{cm}^{2}$. This is also true for etched scratches (see both blue lines, solid or dotted). For scratch widths larger than $25 \mu \mathrm{m}$, etching of the substrate before coating seems to reduce the damage threshold as indicated by the red and green dotted lines. In both cases, the damage threshold decreases to $30 \mathrm{~J} / \mathrm{cm}^{2}$. Etching, however, does not affect the behavior of the scratches less than $20 \mu \mathrm{m}$ in width (see blue dotted line). 

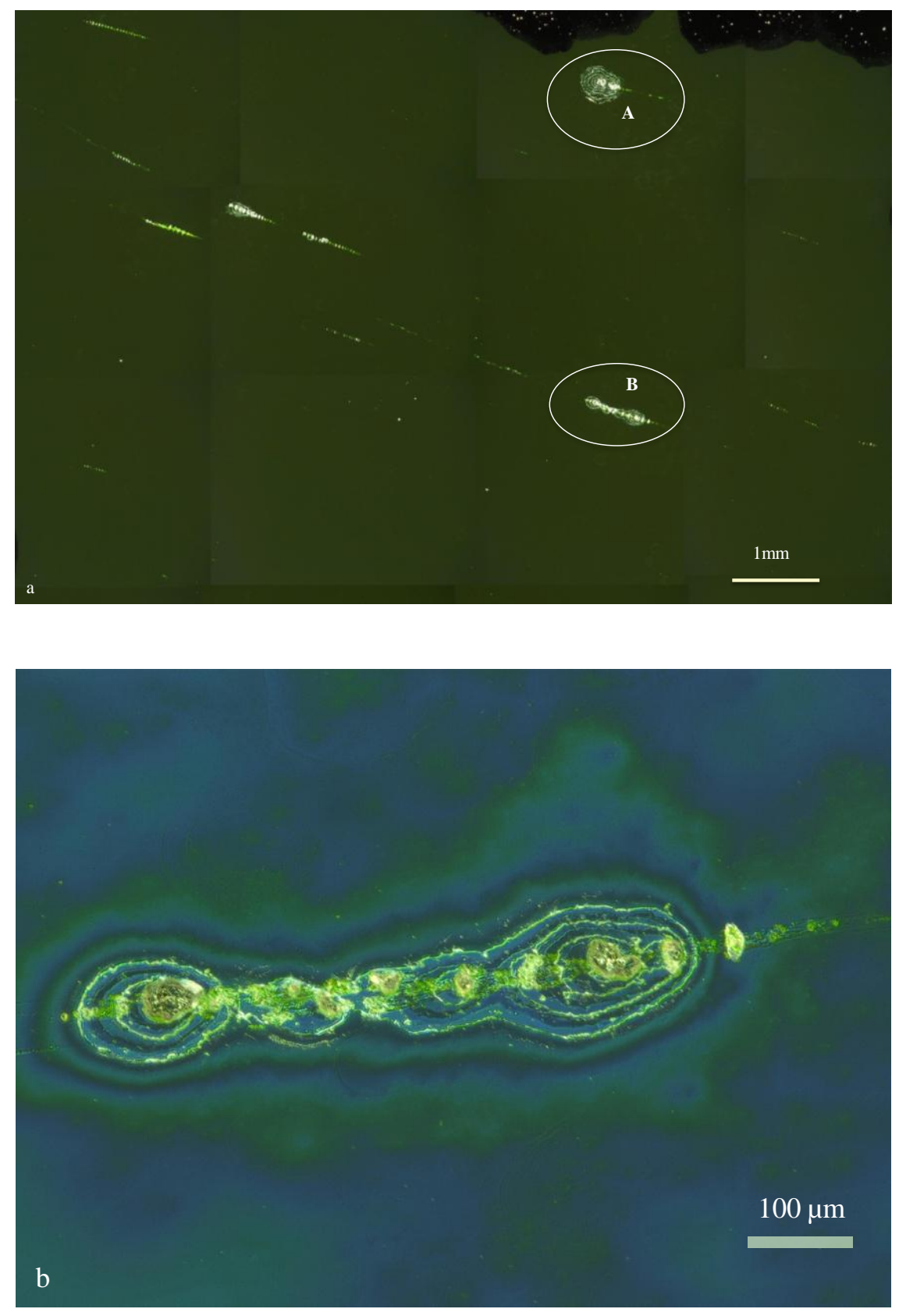

Figure 3. (a) Optical microscope image to show examples of damage sites on the multilayer coating where substrate scratches reside. (b) Optical microscope image of enlarged site B in image (a). 


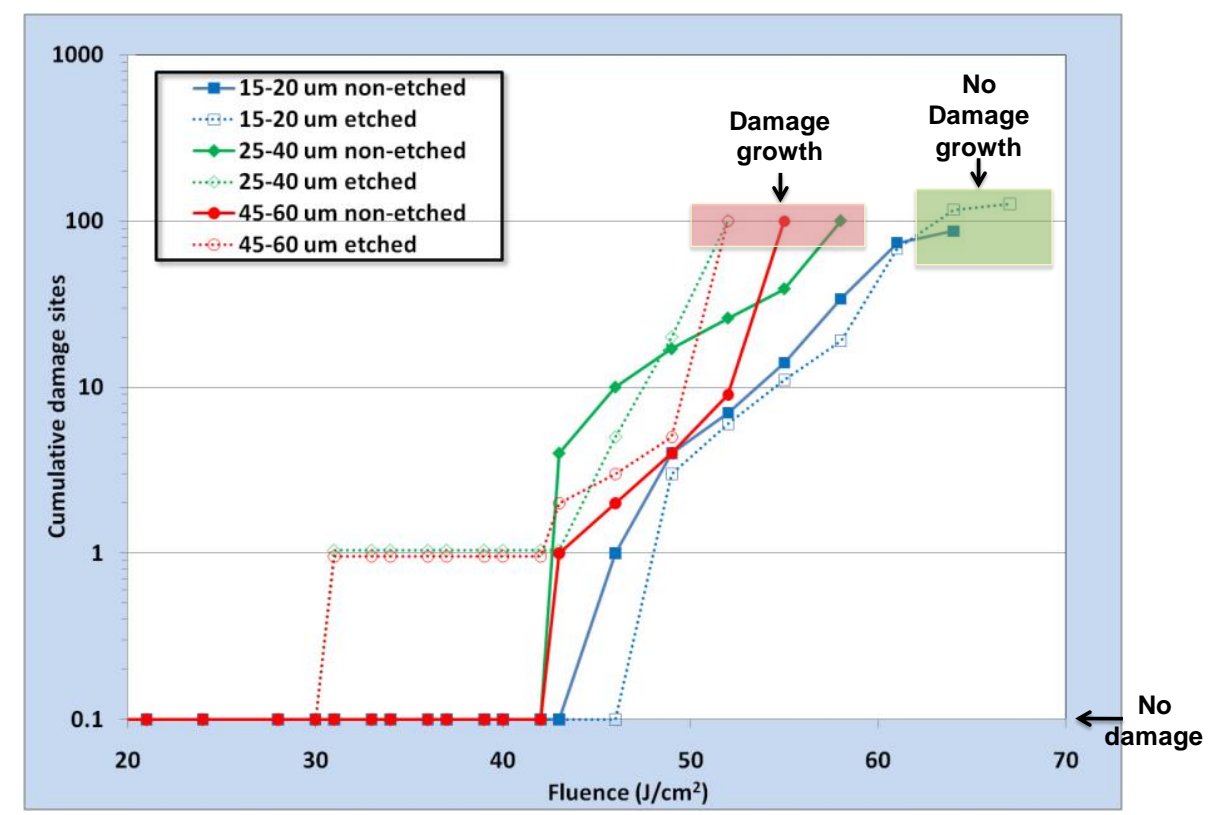

Figure 4. Laser damage initiation and growth threshold for HR multilayer coating on BK7 substrate with surface scratches of different width.

There are many possible explanations of why substrate scratches lead to multilayer coating damage upon laser exposure. Coatings are slightly absorptive so in regions of the coating without macroscopic defects, the intrinsic damage threshold is limited by the electric-field induced thermal breakdown of the coating materials. Earlier studies have shown that electrical field intensification within multilayer layer high reflector interference coatings can occur due to embedded nodular defects or defective pits[12,15]. Electric field intensification within fused silica cracks has also been attributed to reduced laser damage resistance [16]. To elucidate if the coating distorted by substrate scratches can lead to electrical field intensification within the dielectric multilayer, we first collect the crosssectional image of the hafnia-silica multilayer coating on top of a substrate etched sleek (Figure 5 (a), and then estimate the electrical-field distribution of the coating using the aforementioned simulation code. The distribution output of the electrical field intensity $|\mathrm{E}|^{2}$ is plotted in Figure 5 (b). The color scale indicates the electrical field strength. As can be seen in the Figure, the higher electrical-field intensifications are located in the top layer of the coating. The maximum electric-field intensification within the distorted coating due to the existence of the small sleek in the substrate is 4 indicating the scratch would damage at one quarter of the damage threshold of a defect free region of the coating. The amplification of the electrical field within the coating can be attributed to the geometry of the modified layer which acts as a lens to focus the beam to the upper region of the coating. It is apparent that the modification of the coating final geometry strongly depends on the shape of the scratch on the substrate. Since etching can alter the morphology of scratches by increasing the scratch width, the wider scratch will result in a larger area of the coating that modulates the electric field which may lead to a higher magnitude intensification. The direct impact of such effect would be the reduction of damage resistance to laser irradiation.

\section{SUMMARY}

Through experiment and simulation, we find that morphological details of substrate scratches dictate the modulation of a high reflector multilayer geometry which can lead to electrical-field amplification and reduced laser damage resistance. Unlike transmissive fused silica optics, etching of the substrate before multilayer coating degrades rather than improves the laser damage resistance indicating the damage mechanism for scratches on a multi-layer substrate is very different than that for scratches in transmissive fused silica. Finally, scratches of width smaller than $20 \mu \mathrm{m}$ do not generate growing or propagating damage sites over the range of fluences tested in this study. 

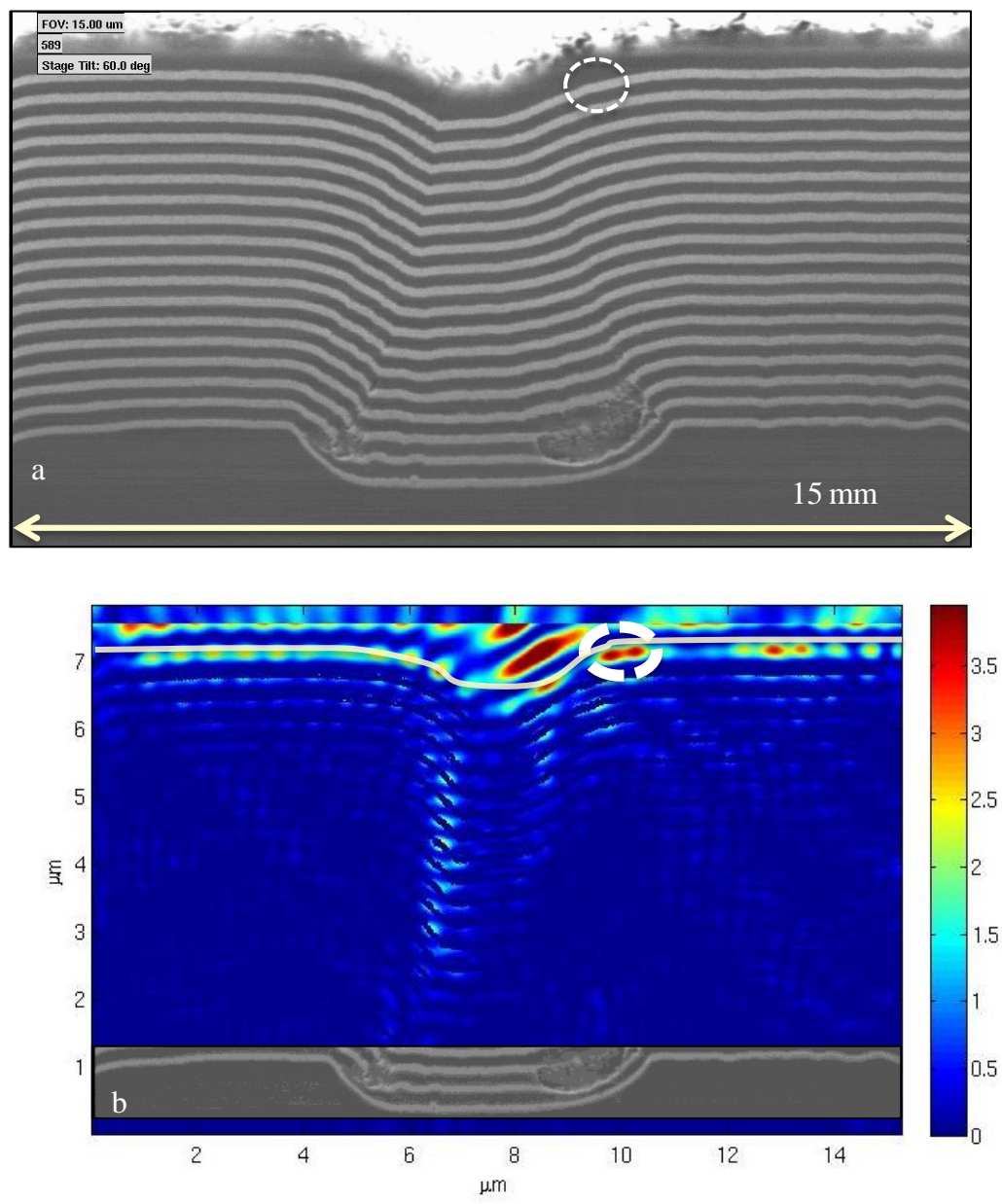

Figure 5. (a) SEM image to show the cross-sectional view of high reflector multilayer coating on top of a sleek scratch on the substrate surface. The coating changes its morphology accordingly while trying to recover its flatness. (b) Distribution of electrical-field intensification within the distorted coating due to the existence of sleek scratch on the substrate surface as shown in (a).

\section{ACKNOWLEDGEMENT}

The authors would like to thank Michael Thomas at the Spica Technologies Inc. for performing the laser-induced damage testing. This work was performed under the auspices of the U.S. Department of Energy by Lawrence Livermore National Laboratory under Contract DE-AC52-07NA27344. This document was prepared as an account of work sponsored by an agency of the United States government. Neither the United States government nor Lawrence Livermore National Security, LLC, nor any of their employees makes any warranty, expressed or implied, or assumes any legal liability or responsibility for the accuracy, completeness, or usefulness of any information, apparatus, product, or process disclosed, or represents that its use would not infringe privately owned rights. Reference herein to any specific commercial product, process, or service by trade name, trademark, manufacturer, or otherwise does not necessarily constitute or imply its endorsement, recommendation, or favoring by the United States government or Lawrence Livermore National Security, LLC. The views and opinions of authors expressed 
herein do not necessarily state or reflect those of the United States government or Lawrence Livermore National Security, LLC, and shall not be used for advertising or product endorsement purposes.

*Address correspondence to S. Roger Qiu, Lawrence Livermore National Laboratory, 7000 East Avenue, L-491, Livermore, CA 94551. Email: qiu2@1lnl.gov. Phone: (925) 422-1636.

\section{REFERENCES}

1. J. DiJon, T. Poiroux, and C. Desrumaux, "Nano absorbing centers: a key point in the laser damage of thin films," Proc. SPIE 2966, 315-325 (1997).

2. F. Y. Genin and C. J. Stolz, "Morphologies of laser-induced damage in hafnia-silica multilayer mirror and polarizer coatings," Proc. SPIE 2870, 439-448 (1996).

3. J. DiJon, P. Garrec, N. Kaiser, and U. B. Schallenberg, "Influence of substrate cleaning on LIDT of $355 \mathrm{~nm}$ HR coatings," Proc. SPIE 2966, 178-186 (1997).

4. C. J. Stolz, J. Adams, M. D. Shirk, M. A. Norton, and T. L. Weiland, "Engineering meter-scale laser resistant coatings for the near IR," Proc. SPIE 5963, 59630Y-59631 (2005).

5. P. E. Miller, T. I. Suratwala, J. D. Bude, T. A. Laurence, N. Shen, W. A. Steele, M. D. Feit, J. A. Menapace, and L. L. Wong, "Laser damage precursors in fused silica," in (SPIE, 2009), 75040X.

6. P. E. Miller, J. D. Bude, T. I. Suratwala, N. Shen, T. A. Laurence, W. A. Steele, J. A. Menapace, M. D. Feit, and L. L. Wong, "Fracture-induced subbandgap absorption as a precursor to optical damage on fused silica surfaces," Optics Lett. 35, 2702 (2010).

7. M. R. Borden, J. A. Folta, C. J. Stolz, J. R. Taylor, J. E. Wolfe, A. J. Griffin, and M. D. Thomas, "Improved method for laser damage testing coated optics," Proc. SPIE 5991, A9912-A9912 (2005).

8. T. V. Pistor, "Electromagnetic simulation and modeling with applications in lithography," (University of California, Berkeley, Berkeley, 2001).

9. A. Wong, "Rigorous three-dimensional time domain finite-difference electromagnetic simulation," (University of California, Berkeley, Berkeley, 1994).

10. K. S. Yee, "Numerical Solution of Initial Boundary Value Problems Involving Maxwell's Equations in Isotropic Media," IEEE Trans. Antennas Propag 14, 302-307 (1996).

11. J. P. Berenger, "A perfectly matched layer for the absorption of electromagnetic waves," Journal of Computational Physics 114, 185-200 (1994).

12. S. R. Qiu, J. E. Wolfe, A. M. Monterrosa, M. D. Feit, T. V. Pistor, and C. J. Stolz, "Searching for optimal mitigation geometries for multilayer high reflector coatings," Appl. Opt. (2011).

13. S. R. Qiu, J. E. Wolfe, A. M. Monterrosa, M. D. Feit, T. V. Pistor, and C. J. Stolz, "Modeling of light intensification by conical pits within multilayer high reflector coatings," Proc. SPIE 7504, 75040M-75041 (2009).

14. P. K. Weber, G. A. Graham, N. E. Teslich, W. M. Chan, S. Ghosal, T. J. Leighton, and K. E. Wheeler, "NanoSIMS imaging of Bacillus spores sectioned by focused ion beam," J. Microsc.-Oxf. 238, 189-199 (2010).

15. C. J. Stolz, M. D. Feit, and T. V. Pistor, "Laser intensification by spherical inclusions embedded within multilayer coatings," Appl. Opt. 45, 1594-1601 (2006).

16. F. Y. Genin, A. Salleo, T. V. Pistor, and L. L. Chase, "Role of light intensification by cracks in optical breakdown on surfaces," Journal of the Optical Society of America a-Optics Image Science and Vision 18, 2607-2616 (2001). 